\title{
Toward industrialization: Supporting the manufacturing processes of superconducting cavities at DESY
}

\author{
J. Bürger, J.A. Dammann, L. Hagge, J. Iversen, A. Matheisen, W. Singer * \\ Deutsches Elektronen-Synchrotron (DESY), Notkestrasse 85, 22607 Hamburg, Germany
}

Abstract

Manufacturing high-gradient superconducting cavities for future accelerators requires detailed knowledge of the entire production process. This knowledge has to be transferred from the laboratories, which are developing the process, to industry in order to achieve reproducible results in the industrial production of large numbers of cavities. The paper introduces DESY's approach to process industrialization based on the use of an engineering data management system (EDMS).

(C) 2006 Published by Elsevier B.V.

Keywords: Superconducting RF cavities; Design; Manufacturing; Conditioning

\section{Introduction}

About 1000 high-gradient s.c. cavities have to be produced by industry for the proposed European XFEL. The production process includes cavity fabrication and subsequent preparation activities for performance optimization (e.g., [1,2]). A series of 30 cavities which are presently under production at DESY in collaboration with industry shall be used to study the general requirements on process control and documentation tools.

For the preparation of the XFEL project, an engineering data management system (EDMS) has been introduced at DESY as a central information management platform. The EDMS is based on a commercial system which has been adapted to DESY's needs. It is used to support the cavity production processes, including external manufacturers, to enable quality management and to prepare knowledge transfer to industry. The EDMS also manages technical project documentation and supports other planning activities of the XFEL [3].

\footnotetext{
${ }^{*}$ Corresponding author. Tel.: +494089982775; fax: +494089981970. E-mail address: waldemar.singer@desy.de (W. Singer).
}

\section{Process industrialization}

Process know-how includes task definitions, the task sequencing and the resulting process data. Transferring a process from DESY to an industrial company is foreseen to happen in three phases (cf. Fig. 1(a)-(c)).

First, a process has to be defined, established and optimized. The process steps are documented as so-called "work packages" (WP), which contain work instructions and templates for recording the results. Each WP is performed by a specific process team (a).

Next, the WPs are entered into the EDMS, which then takes over the process coordination (b). Each process is initiated by the process manager (e.g., when a new cavity is received). The EDMS is then sending the work instructions to the responsible process teams in the correct sequencing. The process teams perform the required work and record the results in the EDMS. Each WP result is inspected and approved by the process manager before the next WP is issued by the EDMS. The WP specifications in the EDMS are evolved with growing process experience.

As the EDMS is fully web-based, teams in remote locations can easily be included into the procedure. This way, process steps can be sourced out, and external collaborators and companies can be gradually integrated as the pro- 

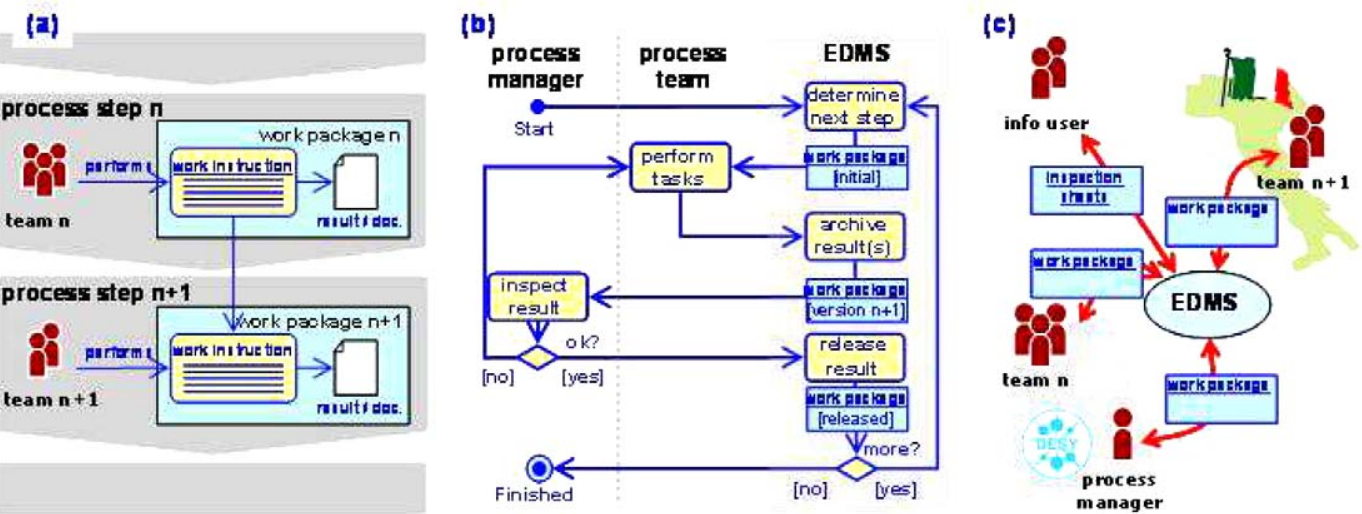

Fig. 1. Three phases of industrializing the cavity preparation process: (a) process analysis and definition, (b) process coordination by an engineering data management system (EDMS) and (c) process distribution.

58 cess matures (c). In the end the process can be fully exe-

59 cuted in industry, while DESY can stay involved in the pro-

60 cess coordination and supervision by taking the role of the

61 process manager.

\section{3. EDMS operation principle}

An EDMS is an information system for managing the information and coordinating the processes of the entire product life cycle. An EDMS offers work flows, databases and user interfaces for supporting activities such as specification, mechanical (3D CAD) design, manufacturing, conditioning or installation.

An EDMS provides so-called "views" which contain the relevant information for the different life cycle activities, and it establishes traceability between these views (cf. Fig. 2). Each view is organized around a hierarchical structure whose elements are associated with the necessary process data. For example, in the EDMS, a cavity corresponds to a "part". The design view contains the hierarchical part breakdown structure (PBS) in which the parts are associated with their 3D CAD models, drawings, FEM calculations and further documents. For manufactured or "serialized" parts it can later be determined from which version of the design data they have been created by fol- lowing the "is-serialized-from" relation. The mechanism 81 is repeated for all the views in an EDMS.

\section{EDMS-supported cavity production}

DESY has implemented design, manufacturing and conditioning views in its EDMS for supporting the production of s.c. cavities for the TTF/VUV-FEL facility.

\subsection{Design view}

The design view defines the cavity's geometry and properties and is the source for simulations, calculations and manufacturing. Fig. 3 shows the mechanical design of a cavity in a 3D CAD system and an excerpt of its PBS as seen in the EDMS. CAD users can access and modify the CAD models and the PBS through the CAD system, while other users can access and view the PBS and its associated $3 \mathrm{D}$ visualization models, drawings and documents through the EDMS web client.

\subsection{Manufacturing view}

Fig. 4 shows the manufacturing view of a 9-cell cavity. 98 For every individual cavity, so-called serialized cavities 99

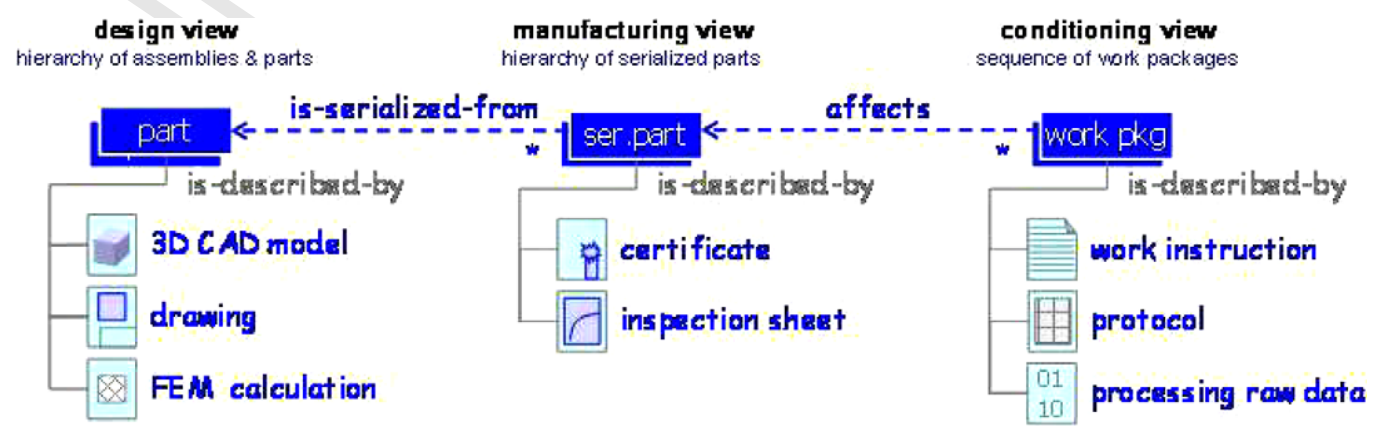

Fig. 2. Organization of information in an engineering data management system (EDMS). 


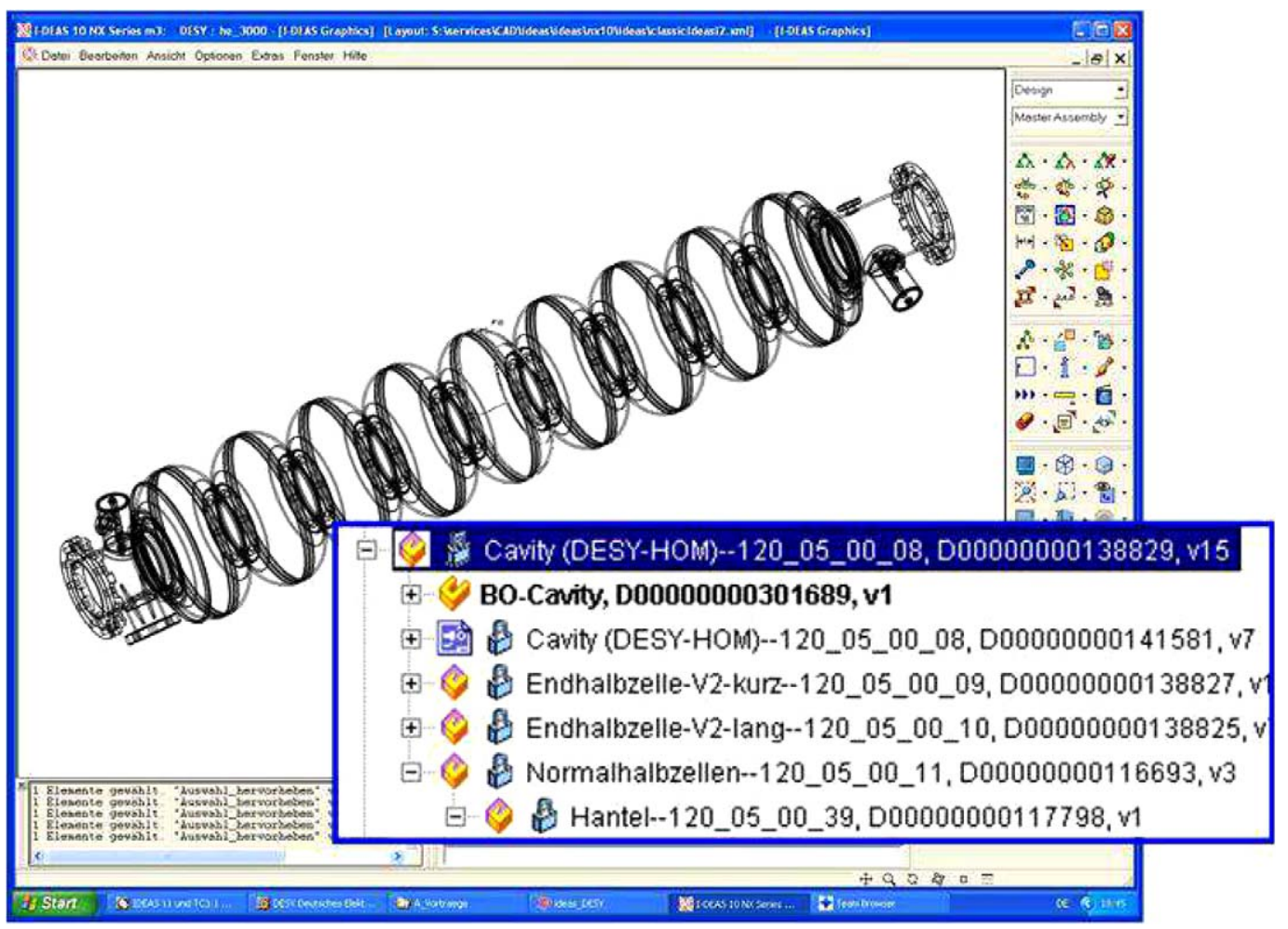

Fig. 3. Design view of a cavity in a 3D CAD system and corresponding part breakdown structure in the EDMS.

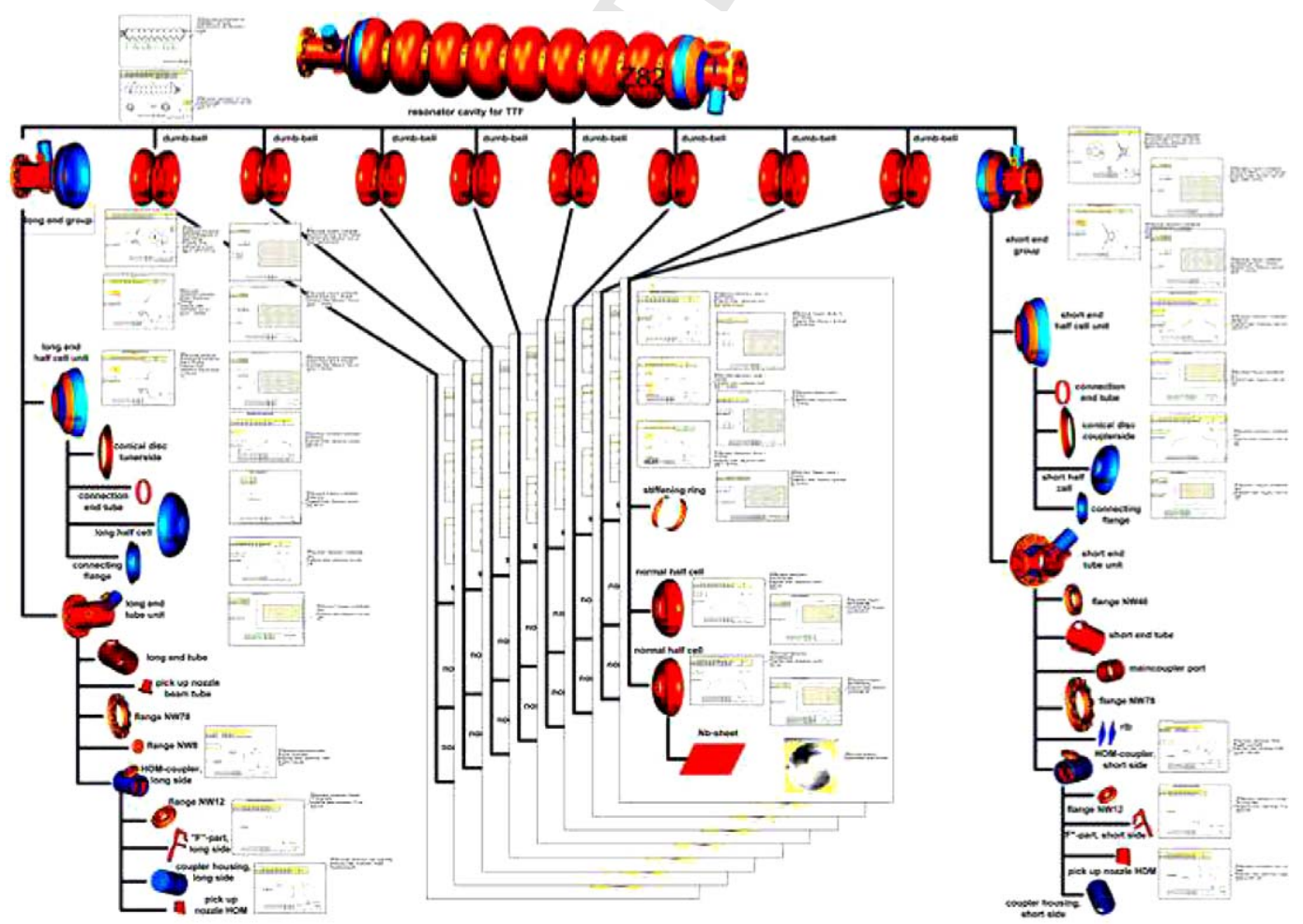

Fig. 4. Manufacturing view of a cavity in the EDMS, showing the serialized part breakdown structure with the associated inspection sheets for quality control.

100 are created in the EDMS. They are built along serialized 101 PBSs (sPBS): while the PBS contains, e.g., one entry for 102 a dumb-bell, which is re-used eight times in the cavity model, a sPBS contains eight entries carrying the serial 103 numbers of the eight manufactured individual dumb-bells 104 which are used in the specific cavity. They are associated 
106 with manufacturing certificates and inspection sheets. The 107 XFEL requires ca. 1000 cavities which will contain about 10870,000 serialized parts and relate to more than 200,000 109 QA documents.

\section{4.3. Conditioning view}

111 Cavities are treated according to a specific preparation 112 procedure for optimizing the rf performance. It consists 113 of 56 defined work packages, including e.g., polishing, bak114 ing and cleaning, which have been defined in and are coor115 dinated by the EDMS according to Fig. 1. Teams receive 116 their work instructions for specific cavities in the EDMS, 117 and they can access the process documentation and get 118 an overview of the current process status using the system.

119 The results of the different conditioning steps are 120 recorded in spread sheets and database entries which are 121 kept the EDMS. Selected data from these records is auto122 matically exported to a separate quality management data123 base for conducting further statistical analysis.

\section{5. Summary and outlook}

125 So far, the EDMS has been used for the production of 126 more than 40 cavities. The initial process definition and 127 the EDMS-based process coordination have increased the 128 efficiency of the preparation process at DESY and reduced 129 the required resources. For keeping track of the cavities growing amount of process records, which are necessary for optimizing the production process, the EDMS has become indispensable.

In its present customization, the EDMS at DESY is well-supporting the needs of the cavity production for the TTF/VUV-FEL facility. It is storing the entire cavity documentation and it is coordinating the cavity preparation procedure. Furthermore, the EDMS-based process definition can be adapted with reasonable effort and in a controlled way as new experience is gained.

In ongoing work, external manufacturers are being connected to the EDMS, enabling DESY to perform quality control activities and to access process documentation already during early stages of the production process.

The available process documentation is an essential foundation for transferring the process to industry, as it is required for building the XFEL. Preparations have been started to extend the process support to the subsequent assembly of the cryomodule, leading to ca. 50 additional work packages per cavity under control of the EDMS, and to cover further components like e.g., couplers and magnets.

\section{References}

[1] B. Aune et al., Phys. Rev. ST Accel. Beams 3 (2000) 092001.

[2] A. Matheisen et al., Proc. PAC'05, TPPT055, Knoxville, May 2005.

[3] J. Bürger et al., Proc. PAC'05, RPPT005, Knoxville, May 2005. 